\title{
Reference Information
}

\section{J. Appl. Cryst. (1970). 3, 549}

A system of programs for generalized superpositions.* By C.R. HubBard and R. A. JACobson, Institute for Atomic Research and Department of Chemistry, Iowa State University, Ames, Iowa 50010, U.S.A.

(Received 8 July 1970)

From a generalized definition of a superposition map, a system of programs has been written to perform the superposition of several Patterson and/or pseudo electron density maps.

The superposition technique (Buerger, 1959) was introduced to locate one image of an $\mathrm{N}$-atom structure by deconvolution of the $N$ images of the Patterson map. In practice, this process consists of performing a superposition of several shifted Patterson maps on an unshifted Patterson map. Each shift vector must be a vector from a given atom to any of the other $N-1$ atoms in the same image. Generalization of the base map to include symmetry maps (Mighell \& Jacobson, 1963; Simpson \& Dobrott, 1965) and pseudo electron density maps extends the usefulness of the superposition technique. A generalized definition of a superposition map is

$$
\begin{gathered}
S(\mathbf{u})=\operatorname{MIN}\left\{\operatorname{MAP} 1(\mathbf{u}), w_{1}^{*} \operatorname{MAP} 2\left(\mathbf{u}-\mathbf{s}_{1},\right.\right. \\
\left.w_{2} * \operatorname{MAP} 2\left(\mathbf{u}-\mathbf{s}_{2}\right), \ldots\right\},
\end{gathered}
$$

where MAP2 $=$ Patterson map, MAP1 = Patterson, symmetry, or pseudo electron density map, $\mathbf{s}_{i}=$ shift vector, and $w_{i}=$ weighting factor.

A system of programs to eficiently calculate the superposition map $S(\mathbf{u})$ has been written in FORTRAN IV for the IBM 360/65 computer and consists of three programs: $G E N, S Y M M$, and $A L S$. The first program is used to generate the data set of appropriate format for the other two programs using the output of a Patterson program (Rodgers \& Jacobson, 1969). The second program, SYMM, is used if a symmetry map is to be calculated. The third program, $A L S$, performs the superposition and was written with four requirements in mind: (1) small computer core

* Work performed in the Ames Laboratory of the U.S. Atomic Energy Commission. Contribution No. 2812. allocation, (2) high speed excution, (3) generalized superpositions using the minimum function, and (4) weighting, origin shift, and multiple superposition options.

To satisfy these four requirements, $A L S$ calculates all superpositions for a given layer of the resultant superposition map before proceeding to the next layer. With this technique, storage for only two-dimensional arrays is required. Storage requirements are further reduced by using two-byte words for each point in the base and Patterson maps. The symmetry unique regions of both maps are stored on magnetic disk packs in the direct access mode; each layer is randomly accessible. Input delays are reduced by appropriate buffering and use of the direct access FIND statement which permits overlapping of disk access and computation. The calculation of eight superpositions of a monoclinic Patterson on a symmetry map of grid $31 * 43 * 23$ required $64 \mathrm{~K}$ bytes storage and 100 seconds CPU time on our computer.

Card decks and a descriptive report (IS-2210) may be obtained on request from the authors.

\section{References}

Buerger, M. J. (1959). Vector Space. New York: John Wiley.

Mighell, A. D. \& Jacobson, R. A. (1963). Acta Cryst. 16, 443.

RODGERs, J. \& JACOBSON, R. A. (1969). A General Fourier Program in PLI, IS-2155, Ames Laboratory, Ames, Iowa 50010.

Simpson, P. G., Dobrott, R. D. \& Lipscomb, W. N. (1965). Acta Cryst. 18, 169.

\section{Book Reviews}

Works intended for notice in this column should be sent direct to the Book-Review Editor (M.M.Woolfson, Physics Department, University of York, Heslington. York YO1 5DD, England). As far as practicable books will be reviewed in a country different from that of publication.

Technical metallurgy. By D. R. Cliffe. Pp. v+414. London: Arnold, 1968. Price $45 s$.

Physical metallurgy has seen fast progress in the last few decades. It is only with considerable delay that this progress has been transmitted to the curricula for students of mechanical engineering and industrial metallurgy. The textbook written by D. R. Cliffe is an effort to show the many ways in which principles of physical metallurgy can become fruitful for engineering application. One hopes that courses of this type will replace the descriptive representation of data which traditionally has been predominant in the teaching of technological subjects.

Many of the 17 chapters bear familiar headlines such as 'defects in metal crystals', 'deformation', 'heat treatment' and 'constitution' but there are also chapters such as 'vacuum techniques', 'metal joining', 'temperature measurement and control' which one does not usually find in books of this kind. There is even an appendix on computers.

This great number of topics treated has prevented the author from going into depth. The book must therefore be considered as a mere introduction to the field, to be supplemented by further studies.

H. P. STÜWE

Institut für Werkstoffkunde und Herstellungsverfahren

Technische Hochschule Braunschweig

33 Braunschweig

Pockelstrasse 4

Germany 